\title{
PREVALENT BACTERIA AND THEIR SENSITIVITY AND RESISTANCE PATTERN TO ANTIBIOTICS: A STUDY IN DHAKA MEDICAL COLLEGE HOSPITAL.
}

\author{
HOSSAIN MZ ${ }^{1}$, NAHER A ${ }^{2}$, HASAN P ${ }^{3}$, MOZAZFIA KT ${ }^{4}$, TASNIM H ${ }^{5}$, FERDUSH $Z^{6}$, TOWHID KMS ${ }^{7}$, \\ IMRAN MAA ${ }^{8}$
}

\begin{abstract}
Background and rationale: Antibiotic resistance is a global problem. Many factors are complexly related to the issue in multiple dimensions. Bangladesh is right in the middle of this great calamity, and is seeing the rise in resistant strains of several bacteria. Very sadly, the prevalent malpractice of abusing antibiotics in Bangladesh contributes to add complexity to the danger which may prove to be possibly the greatest threat humans have ever faced. There is much scarcity of medical literature in Bangladesh, on the antibiotic sensitivity pattern and prevalent microorganisms. Moreover, antibiotic sensitivity pattern changes over time and place. Again, most of the studies done in Bangladesh, concentrate on a single disease, pathogen, or specimen. This study attempts to see the prevalent microorganisms and the antibiotic sensitivity pattern in multiple types of specimens collected from Dhaka Medical College Hospital. This study also attempts to establish a way of presentation of the relevant findings which can be used in future to ensure easy comparability and contrasting of findings.

Methods: The specimens were collected from the adult patients (age $>12$ years) admitted in the Internal Medicine ward of Dhaka Medical College Hospital, Dhaka, over a period of 6 months. The sampling technique was consecutive sampling method. Specimens which were culture positive, were only included in the study for analysis. Multiple specimens were taken.

Results: S. aureus was 100\% sensitive to amikacin, moxifloxacin, imipenem, meropenem, piperacillin+tazobactum combination, vancomycin, doxycycline, tetracycline, tigecycline, nitrofurantoin, azactum, linezolid and 100\% resistant to cefixime. Enterobacter was 100\% sensitive to penicillin, amikacin, gentamicin, netilmicin, doxycycline, tetracycline, tigecycline and $100 \%$ resistant to cefixime, ceftazidime, ceftriaxone, cefepime, cotrimoxazole, levofloxacin, vancomycin. E. coli was $100 \%$ sensitive to imipenem, meropenem, vancomycin, tigecycline and 100\% resistant to mecillinam, aztreonam. Klebsiella was 100\% sensitive to flucloxacillin, colistin, vancomycin, tigecycline, linezolid and 100\% resistant to nalidixic acid. Proteus was $100 \%$ sensitive to cephradine, cefoxitin, cefixime, ceftazidime, ceftriaxone, cefepime, cotrimoxazole, amikacin, ciprofloxacin, imipenem, meropenem, netilmicin, piperacillin+tazobactum combination, tetracycline, tigecycline, azithromycin, azactum and 100\% resistant to doxycycline, tetracycline, chloramphenicol and cefuroxime. Pseudomonas was 100\% sensitive only to amikacin, netilmicin, and 100\% resistant to cefixime, ceftazidime, ceftriaxone, cefepime, cotrimoxazole, gentamicin, ciprofloxacin, levofloxacin, imipenem, meropenem, doxycline, tetracycline, chloramphenicol. Salmonella typhi was 100\% sensitive to amoxicillin, cefoxitin, cefixime, ceftriaxone, cefepime, cotrimoxazole, amikacin, netilmicin, azithromycin, chloramphenicol, azactum and 100\% resistant to cephradine, doxycycline, tetracycline, nalidixic acid. MRSA was 100\% sensitive to imipenem, vancomycin, teicoplanin, nitrofurantoin, linezolid and $100 \%$ resistant to cefpirome, cefoxitin, ceftazidime, cotrimoxazole, clindamycin, gentamicin, ciprofloxacin, netilmicin, tetracycline, clarithromycin. Acinetobacter was $100 \%$ sensitive to penicillin, cefuroxime, colistin, piperacillin+tazobactum combination, tigecycline, chloramphenicol and $100 \%$ resistant to cefixime, nalidixic acid. Citrobacter freundii was $100 \%$ sensitive to ceftazidime, ceftriaxone, cotrimoxazole, amikacin, gentamicin, ciprofloxacin, levofloxacin, imipenem, meropenem, netilmicin, nalidixic acid and $100 \%$ resistant to ampicillin, cefixime, nitrofurantoin.
\end{abstract}

Conclusion: More and more antibiotics are becoming ineffective due to emergence of resistance. Serious actions should be taken. Awareness should be raised from the policy maker level to the physicians and patients.

Keywords: Antibiotic, Culture sensitivity, Resistance

J Dhaka Med Coll. 2017; 26(1):

$52-64$

1. Dr. Mohammad Zaid Hossain, Associate Professor, Department of Medicine, Dhaka Medical College, Dhaka.

2. Dr. Akhtarun Naher, Professor, Department of Microbiology, Sir Salimullah Medical College, Dhaka.

3. Dr. Pratyay Hasan, Indoor Medical Officer, Department of Medicine, Dhaka Medical College Hospital, Dhaka.

4. Dr. Kazi Tuba-E Mozazfia, Assistant Registrar, Department of Medicine, Shaheed Suhrawardy Medical College, Dhaka.

5. Dr. Homyra Tasnim, Intern Doctor, Department of Medicine, Dhaka Medical College Hospital, Dhaka.

6. Dr. Zannatul Ferdush, Intern Doctor, Department of Medicine, Dhaka Medical College Hospital, Dhaka.

7. Dr. Kazi Md. Saleheen Towhid, Indoor Medical Officer, Department of Medicine, Dhaka Medical College Hospital, Dhaka.

8. Dr. Md. Abdullah Al Imran, Indoor Medical Officer, Department of Medicine, Dhaka Medical College Hospital, Dhaka Correspondence: Dr. Mohammad Zaid Hossain, Associate Professor, Department of Medicine, Dhaka Medical College, Dhaka. Email: zhvalentino@gmail.com

Received: 03 January 2017

Accepted: 01 March 2017 


\section{Introduction}

Antibiotic resistance is a global problem. ${ }^{1-15}$ The issue has always been a major concern, but now it has probably become more pressing than ever before. Many factors are complexly related to the issue in multiple dimensions. There are many theories concerning the genesis of the phenomenon, including gross lack of awareness, inaction, ${ }^{16}$ excess use of antibiotics in the field of agriculture or aquaculture, ${ }^{11}$ emergence of new mechanisms, ${ }^{17-20}$ etc. Though many opinions exist about how this danger, which is described as a global pandemic ${ }^{12}$ or a worldwide calamity ${ }^{1}$ came into existence, it is not difficult to reach a universal consensus on the grave consequences that awaits the whole human race, caused by this single reason. Developing world is not exempted, rather more in the danger. ${ }^{5,15,21}$ Bangladesh is also right in the middle of this great calamity and is seeing the rise in resistant strains of several bacteria. ${ }^{22-}$ ${ }^{25}$ It is noteworthy, that the causes of antibiotic resistance are often postulated to be its misuse and abuse. ${ }^{15,24,26}$ Bangladesh has become a major field of antibiotic misuse and abuse. ${ }^{26-}$ 30 Very sadly, this prevalent malpractice of abusing antibiotics in Bangladesh contributes to add complexity to the danger which may prove to be possibly the greatest threat humans have ever faced. There is much scarcity of medical literature in Bangladesh, on the antibiotic sensitivity pattern and prevalent microorganisms. Moreover, antibiotic sensitivity pattern changes over time and place. ${ }^{31-36}$ Which is why it is an imperative, especially in today's age of antibiotic resistance, to continuously monitor and survey the prevalence of different microorganisms, antibiotic sensitivity pattern and resistance pattern. Again, most of the studies done in Bangladesh, concentrate on a single disease, pathogen, or specimen. ${ }^{37-51}$ But, a single organism can cause different types of infection, at different sites and can cause different diseases. For example, E. coli can cause gastroenteritis, sepsis, UTI, even meningitis. So to get a clearer, broader picture of the prevalent microorganisms and the antibiotic sensitivity pattern, multiple types of specimen should be analyzed together. This study attempts to see the prevalent microorganisms and the antibiotic sensitivity pattern in multiple types of specimens collected from Dhaka Medical College Hospital. This study also attempts to establish a way of presentation of the relevant findings which can be used in future to ensure easy comparability and contrasting of findings.

\section{Materials and methods}

Sampling technique

The specimens were collected from the adult patients (age $>12$ years) admitted in the Internal Medicine ward of Dhaka Medical College Hospital, Dhaka, over a period of 6 months. The sampling technique was consecutive sampling method. Specimens which were culture positive, were only included in the study for analysis. Multiple specimens were taken:

1. Blood

2. Sputum

3. Urine

4. Stool

5. Pus

6. Wound swab

7. High vaginal swab

Only CSF was excluded for convenience. Cultures of the selected specimens were only done when it was indicated by mentioned criteria.

\section{Indication of blood culture}

1. If the patient presented with features suggestive of one of the following ${ }^{52}$

a. Sepsis

b. Endocarditis

c. Osteomyelitis

d. Meningitis

e. Pneumonia

OR,

2. The patient was suffering from pyrexia of unknown origin

\section{Indication of sputum culture}

1. If the patient presented with features suggestive of one of the following 52 
a. Pneumonia

b. Tuberculosis

2. The patient was suffering from pyrexia of unknown origin and presented with productive cough.

\section{Indication of urine culture}

1. If the patient presented with features suggestive of one of the following ${ }^{52}$

a. Pyelonephritis

b. Cystitis

2. The patient was suffering from pyrexia of unknown origin and dysuria or frequency was present.

\section{Indication of stool culture}

1. Enterocolitis was suspected ${ }^{52}$ or,

2. The patient was suffering from pyrexia of unknown origin

\section{Indication of culture of pus}

1. The patient presented with abscess or localized collection of pus

Indication of wound swab culture

1. If the patient presented with infected wound

\section{Indication of high vaginal swab culture}

1. If the female patient presented with abnormal discharge

2. Or, had history of contact with a partner with sexually transmitted diseases (STDs)

\section{Method of blood specimen collection}

Sample of blood was collected from the patient after cleaning the venipuncture site with povidone iodine and $70 \%$ alcohol. Two to three (2-3) $\mathrm{ml}$ of blood was collected aseptically by a gloved hand using a sterile disposable syringe.

\section{Method of urine specimen collection}

Local disinfection of the meatus and adjacent mucosa was done with a non-foaming antiseptic solution; this region then was dried with a sterile swab to avoid mixture of the antiseptic with urine. Contact of the urinary stream with the mucosa was minimized by spreading the labia in females and by pulling back the foreskin in uncircumcised males. The first voided specimen was discarded since the initial urine flushes urethral contaminants. The second, midstream sample was sent to the laboratory.

\section{Method of stool specimen collection}

Stool was collected with all aseptic precautions

Method of pus specimen collection

Aspirated material was sent to laboratory in suitable containers.

Method of wound swab specimen collection The specimen was obtained prior to any dressing or cleaning procedure of the wound. This allowed maximized material obtained and prevented killing of the organism by the use of antiseptics. A sterile swab was used and gently rotated on the area to collect exudate from the wound and then placed into transport medium. Where there was pus, it was collected as much as possible in a sterile syringe or sterile container and send to the laboratory.

\section{Method of high vaginal swab specimen collection}

The patient's labia were open apart with the help of speculum and swab was placed high inside the vaginal canal. The swab specimen was then taken off into the transport tube.

\section{Method of blood culture}

The blood was inoculated in the fan bottle and will be incubated at $37^{\circ} \mathrm{C}$, aerobically in automated blood culture machine (BACTEC). Sub-culture was done on second day on MacConkey's agar, blood agar, and chocolate agar media and was incubated at $37^{\circ} \mathrm{C}$. Growth of bacterial colony was observed, the bacteria was identified by colony morphology and relevant biochemical tests.

\section{Method of sputum culture}

Sputum was examined in the clinical microbiology laboratory by a direct Gram stain of the specimen and by culture at $35^{\circ} \mathrm{C}$ on the following media: (i) $5 \%$ sheep blood agar (SBA) and $5 \%$ chocolate agar in 5\% CO2 in air; (ii) $5 \%$ SBA under anaerobic condition; and (iii) MacConkey medium in air. ${ }^{53}$

\section{Method of urine culture}

The collected urine specimen was inoculated on MacConkey's and Blood Agar media using calibrated platinum loop following standard bacteriological technique and incubated at $37^{\circ}$ C for 24-48 hours. After 24 to 48 hours the plate 
were examined for bacteria. Pure bacterial colony counting 100,000 or more was considered as significant and was subjected to identification based on colony characteristics and biochemical tests.

\section{Method of pus, wound swab, high vaginal swab culture}

The collected specimens were inoculated in suitable media, preferably MacConkey's and Blood agar media following standard bacteriological technique and incubated at $37^{\circ}$ C for 24-48 hours. After 24 to 48 hours the plate were examined for bacteria. Pure bacterial colony counting 100,000 or more was considered as significant and was subjected to identification based on colony characteristics and biochemical tests.

\section{Method of antibiotic susceptibility testing}

The antibiotic susceptibility of the isolated bacteria was done by modified Kirby- Bauer technique (Bauer, 1996) by disc diffusion method. ${ }^{54}$

\section{Statistical analysis}

After collection of information, these data were analyzed using Statistical Package for Social Sciences (SPSS), version 23 (International Business Machines Corporation, IBM, USA). Descriptive statistics were derived. The results are presented by text, tables and charts and graphs here.

\section{Result}

Specimens collected from a total of 110 patients were included in the study. Age and sex distribution of the patients are shown in Figure 1and Figure 2. Most of the patients were female $(59.09 \%)$, the age distribution resembles a normal distribution (Figure 2).

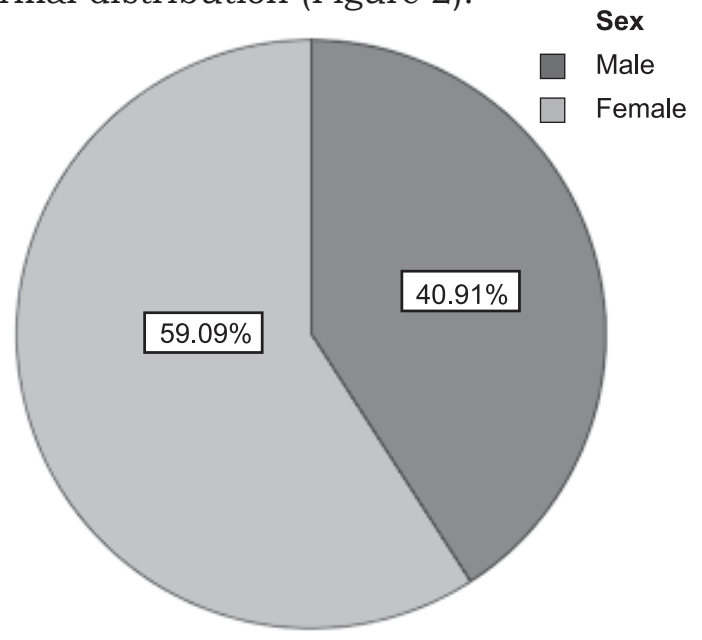

Fig.-1 The sex distribution of the patients

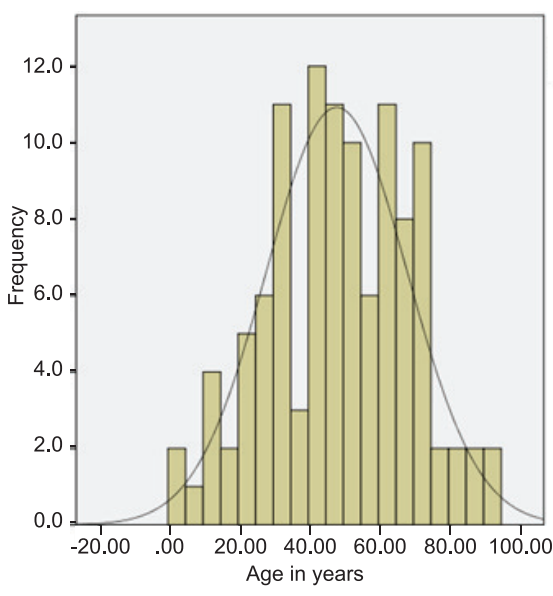

- Normal

Mean $=47.6091$

Std. Dev. $=20.0829$

$\mathrm{N}=110$

Fig.-2: The age distribution

Among the specimens collected (Table 1), most common specimen was urine $(80.9 \%)$ followed by blood $(6.4 \%)$.

Table-I

Different types of specimens, included in the study

\begin{tabular}{lcc}
\hline Specimen & Frequency & Percent \\
\hline Blood & 7 & 6.4 \\
Sputum & 3 & 2.7 \\
Urine & 89 & 80.9 \\
Stool & 1 & .9 \\
Pus & 4 & 3.6 \\
Wound swab & 3 & 2.7 \\
High vaginal swab & 3 & 2.7 \\
\hline Total & 110 & 100.0 \\
\hline
\end{tabular}

Most of the microorganisms isolated were Gram negative (85.5\%).

Table-II

Gram staining pattern of the organisms

\begin{tabular}{lcc}
\hline Gram stain & Frequency & $\%$ \\
\hline Positive & 15 & 13.6 \\
Negative & 94 & 85.5 \\
Others / Mixed & 1 & .9 \\
\hline Total & 110 & 100.0 \\
\hline
\end{tabular}

The microorganisms isolated are shown in Table-III. Most frequently isolated microorganism was E. coli (48.2\%), followed by Klebsiella (17.3\%). The microorganisms isolated in different specimens are shown in 
Table-IV. Again, most frequently encountered microorganism is $E$. coli, in urine. In blood, $E$. coli, and Salmonella typhi both equally were the most frequent microorganisms.

Table-III

Causative organisms

\begin{tabular}{lcc}
\hline Causative organisms & Frequency & Percent \\
\hline Enterococci & 10 & 9.1 \\
Staphylococcus aureus & 6 & 5.5 \\
Enterobacter & 3 & 2.7 \\
Escherichia coli & 53 & 48.2 \\
Klebsiella & 19 & 17.3 \\
Proteus & 3 & 2.7 \\
Pseudomonas & 1 & .9 \\
Salmonella typhi & 3 & 2.7 \\
Staph MRSA & 2 & 1.8 \\
Acinetobacter & 8 & 7.3 \\
Staphylococcus aureus \& & 1 & .9 \\
Pseudomonas & & \\
Citrobacter freundii & 1 & .9 \\
Total & 110 & 100.0 \\
\hline
\end{tabular}

The sensitivity and resistance pattern of different microorganisms to different antibiotics are shown in Table-V.

Enterococci were $100 \%$ sensitive to piperacillin+tazobactum combination, tigecycline, nitrofurantoin and linezolid and $100 \%$ resistant to cefoxitin, cefixime, and moxifloxacin, azithromycin.

S. aureus was $100 \%$ sensitive to amikacin, moxifloxacin, imipenem, meropenem, piperacillin+tazobactum combination, vancomycin, doxycycline, tetracycline, tigecycline, nitrofurantoin, azactum, linezolid and $100 \%$ resistant to cefixime.

Enterobacter was $100 \%$ sensitive to penicillin, amikacin, gentamicin, netilmicin, doxycycline, tetracycline, tigecycline and $100 \%$ resistant to cefixime, ceftazidime, ceftriaxone, cefepime, cotrimoxazole, levofloxacin, vancomycin.

E. coli was $100 \%$ sensitive to imipenem, meropenem, vancomycin, tigecycline and $100 \%$ resistant to mecillinam, aztreonam.

Klebsiella was $100 \%$ sensitive to flucloxacillin, collwastin, vancomycin, tigecycline, linezolid and $100 \%$ resistant to nalidixic acid.

Table-IV

Microorganisms in different specimens

\begin{tabular}{|c|c|c|c|c|c|c|c|c|c|c|c|c|c|c|}
\hline & \multicolumn{2}{|c|}{ Blood } & \multicolumn{2}{|c|}{ Sputum } & \multicolumn{2}{|c|}{ Urine } & \multicolumn{2}{|c|}{ Stool } & \multicolumn{2}{|c|}{ Pus } & \multicolumn{2}{|c|}{$\begin{array}{l}\text { Wound } \\
\text { swab }\end{array}$} & \multicolumn{2}{|c|}{$\begin{array}{c}\text { High } \\
\text { vaginal } \\
\text { swab }\end{array}$} \\
\hline & $\mathrm{N}$ & $\%$ & $\bar{N}$ & $\%$ & $\overline{\mathrm{N}}$ & $\%$ & $\overline{\mathrm{N}}$ & $\%$ & $\mathrm{~N}$ & $\%$ & $\overline{\mathrm{N}}$ & $\%$ & $\bar{N}$ & $\%$ \\
\hline Enterococci & 0 & 0.0 & 0 & 0.0 & 9 & 8.2 & 0 & 0.0 & 1 & 0.9 & 0 & 0.0 & 0 & 0.0 \\
\hline $\begin{array}{l}\text { Staphylococcus } \\
\text { aureus }\end{array}$ & 0 & 0.0 & 0 & 0.0 & 3 & 2.7 & 0 & 0.0 & 1 & 0.9 & 0 & 0.0 & 2 & 1.8 \\
\hline Enterobacter & 0 & 0.0 & 0 & 0.0 & 3 & 2.7 & 0 & 0.0 & 0 & 0.0 & 0 & 0.0 & 0 & 0.0 \\
\hline Escherichia coli & 2 & 1.8 & 0 & 0.0 & 50 & 45.5 & 0 & 0.0 & 0 & 0.0 & 1 & 0.9 & 0 & 0.0 \\
\hline Klebsiella & 1 & 0.9 & 2 & 1.8 & 13 & 11.8 & 0 & 0.0 & 1 & 0.9 & 1 & 0.9 & 1 & 0.9 \\
\hline Proteus & 0 & 0.0 & 0 & 0.0 & 3 & 2.7 & 0 & 0.0 & 0 & 0.0 & 0 & 0.0 & 0 & 0.0 \\
\hline Pseudomonas & 0 & 0.0 & 0 & 0.0 & 0 & 0.0 & 0 & 0.0 & 0 & 0.0 & 1 & 0.9 & 0 & 0.0 \\
\hline Salmonella typhi & 2 & 1.8 & 0 & 0.0 & 0 & 0.0 & 1 & 0.9 & 0 & 0.0 & 0 & 0.0 & 0 & 0.0 \\
\hline Staph $M R S A$ & 1 & 0.9 & 0 & 0.0 & 1 & 0.9 & 0 & 0.0 & 0 & 0.0 & 0 & 0.0 & 0 & 0.0 \\
\hline Acinetobacter & 1 & 0.9 & 1 & 0.9 & 6 & 5.5 & 0 & 0.0 & 0 & 0.0 & 0 & 0.0 & 0 & 0.0 \\
\hline $\begin{array}{l}\text { Staphylococcus } \\
\text { aureus \& } P \\
\text { seudomonas }\end{array}$ & 0 & 0.0 & 0 & 0.0 & 0 & 0.0 & 0 & 0.0 & 1 & 0.9 & 0 & 0.0 & 0 & 0.0 \\
\hline $\begin{array}{l}\text { Citrobacter } \\
\text { freundii }\end{array}$ & 0 & 0.0 & 0 & 0.0 & 1 & 0.9 & 0 & 0.0 & 0 & 0.0 & 0 & 0.0 & 0 & 0.0 \\
\hline
\end{tabular}

$(\mathrm{N}=$ count $)$ 
Table-V

Sensitivity and resistance pattern

\begin{tabular}{|c|c|c|c|c|c|c|c|c|c|c|c|c|}
\hline & & $\begin{array}{c}\text { Entero- } \\
\text { cocci }\end{array}$ & $\begin{array}{c}S . \\
\text { aureus }\end{array}$ & $\begin{array}{l}\text { Entero- } \\
\text { bacter }\end{array}$ & $\begin{array}{l}E . \\
\text { coli }\end{array}$ & $\begin{array}{l}\text { Kleb- } \\
\text { siella }\end{array}$ & $\begin{array}{l}\text { Prote- } \\
\text { us }\end{array}$ & $\begin{array}{c}\text { Pseudo- } \\
\text { monas }\end{array}$ & $\begin{array}{c}\text { Salmo- } \\
\text { nella }\end{array}$ & $\begin{array}{c}M R \\
S A\end{array}$ & $\begin{array}{l}\text { Acineto- } \\
\text { bacter }\end{array}$ & $\begin{array}{c}C . \\
\text { freundii }\end{array}$ \\
\hline & & $\%$ & $\%$ & & $\%$ & $\%$ & $\%$ & $\%$ & $\%$ & $\%$ & $\%$ & $\%$ \\
\hline \multirow[t]{2}{*}{ Penicillin } & $\mathrm{S}$ & 42.9 & 50.0 & 100.0 & 87.5 & 0.0 & 0.0 & 0.0 & 0.0 & 0.0 & 100.0 & 0.0 \\
\hline & $\mathrm{R}$ & 57.1 & 50.0 & 0.0 & 12.5 & 0.0 & 0.0 & 0.0 & 0.0 & 0.0 & 0.0 & 0.0 \\
\hline \multirow[t]{2}{*}{ Ampicillin } & $\mathrm{s}$ & 75.0 & 0.0 & 33.3 & 50.0 & 33.3 & 0.0 & 0.0 & 0.0 & 0.0 & 0.0 & 0.0 \\
\hline & $\mathrm{R}$ & 25.0 & 0.0 & 66.7 & 50.0 & 66.7 & 0.0 & 0.0 & 0.0 & 0.0 & 0.0 & 100.0 \\
\hline \multirow[t]{2}{*}{ Amoxycillin } & $\mathrm{S}$ & 0.0 & 0.0 & 0.0 & 25.0 & 0.0 & 0.0 & 0.0 & 100.0 & 0.0 & 0.0 & 0.0 \\
\hline & $\mathrm{R}$ & 0.0 & 0.0 & 0.0 & 75.0 & 100.0 & 0.0 & 0.0 & 0.0 & 0.0 & 0.0 & 0.0 \\
\hline \multirow{2}{*}{$\begin{array}{l}\text { Amoxycillin }+ \\
\text { Clavulanic Acid }\end{array}$} & $\mathrm{S}$ & 75.0 & 66.7 & 0.0 & 61.5 & 66.7 & 0.0 & 0.0 & 0.0 & 0.0 & 0.0 & 0.0 \\
\hline & $\mathrm{P}$ & 250 & 333 & O 0 & 385 & 333 & 0 & 0 & O & م & م & 0.0 \\
\hline \multirow[t]{2}{*}{ Flucloxacillin } & $\mathrm{S}$ & 0.0 & 0.0 & 0.0 & 0.0 & 100.0 & 0.0 & 0.0 & 0.0 & 0.0 & 0.0 & 0.0 \\
\hline & $\mathrm{R}$ & 0.0 & 0.0 & 0.0 & 0.0 & 0.0 & 0.0 & 0.0 & 0.0 & 0.0 & 0.0 & 0.0 \\
\hline \multirow{2}{*}{ Cloxacillin } & $\mathrm{S}$ & 0.0 & 50.0 & 0.0 & 100.0 & 0.0 & 0.0 & 0.0 & 0.0 & 0.0 & 0.0 & 0.0 \\
\hline & $\mathrm{R}$ & 0.0 & 50.0 & 0.0 & 0.0 & 0.0 & 0.0 & 0.0 & 0.0 & 0.0 & 0.0 & 0.0 \\
\hline \multirow[t]{2}{*}{ Cephradine } & $\mathrm{S}$ & 25.0 & 25.0 & 0.0 & 27.0 & 38.9 & 100.0 & 0.0 & 0.0 & 0.0 & 0.0 & 0.0 \\
\hline & $\mathrm{R}$ & 75.0 & 75.0 & 0.0 & 73.0 & 61.1 & 0.0 & 0.0 & 100.0 & 0.0 & 100.0 & 0.0 \\
\hline \multirow[t]{2}{*}{ Cephalexin } & $\mathrm{S}$ & 0.0 & 0.0 & 0.0 & 0.0 & 0.0 & 0.0 & 0.0 & 0.0 & 0.0 & 0.0 & 0.0 \\
\hline & $\mathrm{R}$ & 0.0 & 0.0 & 0.0 & 0.0 & 0.0 & 0.0 & 0.0 & 0.0 & 0.0 & 0.0 & 0.0 \\
\hline \multirow[t]{2}{*}{ Cefpirome } & $\mathrm{S}$ & 0.0 & 0.0 & 0.0 & 0.0 & 0.0 & 0.0 & 0.0 & 0.0 & 0.0 & 0.0 & 0.0 \\
\hline & $\mathrm{R}$ & 0.0 & 0.0 & 0.0 & 0.0 & 0.0 & 0.0 & 0.0 & 0.0 & 100.0 & 0.0 & 0.0 \\
\hline \multirow[t]{2}{*}{ Cefoxitin } & $\mathrm{S}$ & 0.0 & 75.0 & 0.0 & 66.7 & 50.0 & 100.0 & 0.0 & 100.0 & 0.0 & 40.0 & 0.0 \\
\hline & $\mathrm{R}$ & 100.0 & 25.0 & 0.0 & 33.3 & 50.0 & 0.0 & 0.0 & 0.0 & 100.0 & 60.0 & 0.0 \\
\hline \multirow[t]{2}{*}{ Cefixime } & $\mathrm{S}$ & 0.0 & 0.0 & 0.0 & 31.6 & 47.1 & 100.0 & 0.0 & 100.0 & 0.0 & 0.0 & 0.0 \\
\hline & $\mathrm{R}$ & 100.0 & 100.0 & 100.0 & 68.4 & 52.9 & 0.0 & 100.0 & 0.0 & 0.0 & 100.0 & 100.0 \\
\hline \multirow[t]{2}{*}{ Cefuroxime } & $\mathrm{S}$ & 0.0 & 0.0 & 0.0 & 0.0 & 50.0 & 0.0 & 0.0 & 0.0 & 0.0 & 100.0 & 0.0 \\
\hline & $\mathrm{R}$ & 0.0 & 0.0 & 0.0 & 0.0 & 50.0 & 100.0 & 0.0 & 0.0 & 0.0 & 0.0 & 0.0 \\
\hline \multirow[t]{2}{*}{ Cefotaxime } & $\mathrm{S}$ & 0.0 & 66.7 & 0.0 & 41.7 & 50.0 & 0.0 & 0.0 & 0.0 & 0.0 & 33.3 & 0.0 \\
\hline & $\mathrm{R}$ & 0.0 & 33.3 & 0.0 & 58.3 & 50.0 & 0.0 & 0.0 & 0.0 & 0.0 & 66.7 & 0.0 \\
\hline \multirow[t]{2}{*}{ Ceftazidime } & $\mathrm{S}$ & 50.0 & 33.3 & 0.0 & 57.9 & 76.9 & 100.0 & 0.0 & 100.0 & 0.0 & 42.9 & 100.0 \\
\hline & $\mathrm{R}$ & 50.0 & 66.7 & 100.0 & 42.1 & 23.1 & 0.0 & 100.0 & 0.0 & 100.0 & 57.1 & 0.0 \\
\hline \multirow[t]{2}{*}{ Ceftriaxone } & $\mathrm{S}$ & 20.0 & 25.0 & 0.0 & 31.3 & 47.1 & 100.0 & 0.0 & 100.0 & 0.0 & 50.0 & 100.0 \\
\hline & $\mathrm{R}$ & 80.0 & 75.0 & 100.0 & 68.8 & 52.9 & 0.0 & 100.0 & 0.0 & 0.0 & 50.0 & 0.0 \\
\hline \multirow[t]{2}{*}{ Cefepime } & $\mathrm{S}$ & 25.0 & 60.0 & 0.0 & 66.7 & 82.4 & 100.0 & 0.0 & 100.0 & 0.0 & 50.0 & 0.0 \\
\hline & $\mathrm{R}$ & 75.0 & 40.0 & 100.0 & 33.3 & 17.6 & 0.0 & 100.0 & 0.0 & 0.0 & 50.0 & 0.0 \\
\hline \multirow[t]{2}{*}{ Cotrimoxazole } & $\mathrm{S}$ & 40.0 & 60.0 & 0.0 & 40.9 & 50.0 & 100.0 & 0.0 & 100.0 & 0.0 & 66.7 & 100.0 \\
\hline & $\mathrm{R}$ & 60.0 & 40.0 & 100.0 & 59.1 & 50.0 & 0.0 & 100.0 & 0.0 & 100.0 & 33.3 & 0.0 \\
\hline \multirow[t]{2}{*}{ Clindamycin } & $\mathrm{S}$ & 0.0 & 0.0 & 0.0 & 0.0 & 0.0 & 0.0 & 0.0 & 0.0 & 0.0 & 0.0 & 0.0 \\
\hline & $\mathrm{R}$ & 0.0 & 0.0 & 0.0 & 0.0 & 0.0 & 0.0 & 0.0 & 0.0 & 100.0 & 0.0 & 0.0 \\
\hline \multirow[t]{2}{*}{ Colistin } & $\mathrm{S}$ & 0.0 & 0.0 & 0.0 & 75.0 & 100.0 & 0.0 & 0.0 & 0.0 & 0.0 & 100.0 & 0.0 \\
\hline & $\mathrm{R}$ & 0.0 & 0.0 & 0.0 & 25.0 & 0.0 & 100.0 & 0.0 & 0.0 & 0.0 & 0.0 & 0.0 \\
\hline \multirow[t]{2}{*}{ Amikacin } & $\mathrm{S}$ & 40.0 & 100.0 & 100.0 & 95.6 & 84.2 & 100.0 & 100.0 & 100.0 & 50.0 & 60.0 & 100.0 \\
\hline & $\mathrm{R}$ & 60.0 & 0.0 & 0.0 & 4.4 & 15.8 & 0.0 & 0.0 & 0.0 & 50.0 & 40.0 & 0.0 \\
\hline Gentamicin & $\mathrm{S}$ & 66.7 & 75.0 & 100.0 & 68.6 & 66.7 & 0.0 & 0.0 & 100.0 & 0.0 & 50.0 & 100.0 \\
\hline & $\mathrm{R}$ & 33.3 & 25.0 & 0.0 & 31.4 & 33.3 & 0.0 & 100.0 & 0.0 & 100.0 & 50.0 & 0.0 \\
\hline Tobramycin & $\mathrm{S}$ & 0.0 & 0.0 & 0.0 & 0.0 & 0.0 & 0.0 & 0.0 & 0.0 & 0.0 & 0.0 & 0.0 \\
\hline & $\mathrm{R}$ & 0.0 & 0.0 & 0.0 & 0.0 & 0.0 & 0.0 & 0.0 & 0.0 & 0.0 & 0.0 & 0.0 \\
\hline
\end{tabular}


Table-V (Contd.)

\begin{tabular}{|c|c|c|c|c|c|c|c|c|c|c|c|c|}
\hline & & $\begin{array}{c}\text { Entero- } \\
\text { cocci } \\
\% \\
\end{array}$ & $\begin{array}{c}S . \\
\text { aureus } \\
\%\end{array}$ & $\begin{array}{c}\text { Entero- } \\
\text { bacter } \\
\%\end{array}$ & $\begin{array}{c}E \\
\text { coli } \\
\% \\
\end{array}$ & $\begin{array}{c}\text { Kleb- } \\
\text { siella } \\
\% \\
\end{array}$ & $\begin{array}{c}\text { Prote- } \\
\text { us } \\
\% \\
\end{array}$ & $\begin{array}{c}\text { Pseudo- } \\
\text { monas } \\
\% \\
\end{array}$ & $\begin{array}{c}\text { Salmo- } \\
\text { nella } \\
\% \\
\end{array}$ & $\begin{array}{l}M R \\
S A \\
\% \\
\end{array}$ & $\begin{array}{c}\text { Acineto- } \\
\text { bacter } f i \\
\%\end{array}$ & $\begin{array}{c}C . \\
\text { freundit } \\
\% \\
\end{array}$ \\
\hline \multirow[t]{2}{*}{ Moxifloxacin } & $\mathrm{S}$ & 100.0 & 100.0 & 0.0 & 60.0 & 80.0 & 0.0 & 0.0 & 100.0 & 0.0 & 0.0 & 0.0 \\
\hline & $\mathrm{R}$ & 0.0 & 0.0 & 0.0 & 40.0 & 20.0 & 0.0 & 0.0 & 0.0 & 0.0 & 0.0 & 0.0 \\
\hline \multirow[t]{2}{*}{ Ciprofloxacin } & $\mathrm{S}$ & 83.3 & 60.0 & 33.3 & 42.0 & 68.8 & 100.0 & 0.0 & 100.0 & 0.0 & 42.9 & 100.0 \\
\hline & $\mathrm{R}$ & 16.7 & 40.0 & 66.7 & 58.0 & 31.3 & 0.0 & 100.0 & 0.0 & 100.0 & 57.1 & 0.0 \\
\hline \multirow[t]{2}{*}{ Levofloxacin } & $\mathrm{S}$ & 55.6 & 33.3 & 0.0 & 54.8 & 73.7 & 100.0 & 0.0 & 50.0 & 0.0 & 28.6 & 100.0 \\
\hline & $\mathrm{R}$ & 44.4 & 66.7 & 100.0 & 45.2 & 26.3 & 0.0 & 100.0 & 50.0 & 0.0 & 71.4 & 0.0 \\
\hline \multirow[t]{2}{*}{ Imipenem } & $\mathrm{S}$ & 75.0 & 100.0 & 33.3 & 100.0 & 89.5 & 100.0 & 0.0 & 0.0 & 100.0 & 87.5 & 100.0 \\
\hline & $\mathrm{R}$ & 25.0 & 0.0 & 66.7 & 0.0 & 10.5 & 0.0 & 100.0 & 0.0 & 0.0 & 12.5 & 0.0 \\
\hline \multirow[t]{2}{*}{ Meropenem } & $\mathrm{S}$ & 75.0 & 100.0 & 0.0 & 100.0 & 88.2 & 100.0 & 0.0 & 0.0 & 0.0 & 85.7 & 100.0 \\
\hline & $\mathrm{R}$ & 25.0 & 0.0 & 100.0 & 0.0 & 11.8 & 0.0 & 100.0 & 0.0 & 0.0 & 14.3 & 0.0 \\
\hline \multirow[t]{2}{*}{ Netilmicin } & $\mathrm{S}$ & 60.0 & 100.0 & 100.0 & 88.9 & 72.2 & 100.0 & 100.0 & 100.0 & 0.0 & 50.0 & 100.0 \\
\hline & $\mathrm{R}$ & 40.0 & 0.0 & 0.0 & 11.1 & 27.8 & 0.0 & 0.0 & 0.0 & 100.0 & 50.0 & 0.0 \\
\hline Pipera & $\mathrm{S}$ & 100.0 & 100.0 & 0.0 & 85.7 & 50.0 & 100.0 & 0.0 & 0.0 & 0.0 & 100.0 & 0.0 \\
\hline Tazobactam & $\mathrm{R}$ & 0.0 & 0.0 & 0.0 & 14.3 & 50.0 & 0.0 & 0.0 & 0.0 & 0.0 & 0.0 & 0.0 \\
\hline \multirow[t]{2}{*}{ Vancomycin } & $\mathrm{S}$ & 75.0 & 100.0 & 0.0 & 100.0 & 100.0 & 0.0 & 0.0 & 0.0 & 100.0 & 0.0 & 0.0 \\
\hline & $\mathrm{K}$ & 25.0 & 0.0 & 100.0 & 0.0 & 0.0 & 0.0 & 0.0 & 0.0 & 0.0 & 0.0 & 0.0 \\
\hline \multirow[t]{2}{*}{ Teicoplanin } & $\mathrm{S}$ & 0.0 & 0.0 & 0.0 & 0.0 & 0.0 & 0.0 & 0.0 & 0.0 & 100.0 & 0.0 & 0.0 \\
\hline & $\mathrm{R}$ & 0.0 & 0.0 & 0.0 & 0.0 & 0.0 & 0.0 & 0.0 & 0.0 & 0.0 & 0.0 & 0.0 \\
\hline \multirow[t]{2}{*}{ Doxycycline } & $\mathrm{S}$ & 11.1 & 100.0 & 100.0 & 52.6 & 37.5 & 33.3 & 0.0 & 0.0 & 50.0 & 37.5 & 0.0 \\
\hline & $\mathrm{R}$ & 88.9 & 0.0 & 0.0 & 47.4 & 62.5 & 66.7 & 100.0 & 100.0 & 50.0 & 62.5 & 0.0 \\
\hline \multirow[t]{2}{*}{ Tetracycline } & $\mathrm{S}$ & 20.0 & 100.0 & 100.0 & 51.6 & 55.6 & 100.0 & 0.0 & 0.0 & 0.0 & 50.0 & 0.0 \\
\hline & $\mathrm{R}$ & 80.0 & 0.0 & 0.0 & 48.4 & 44.4 & 0.0 & 100.0 & 100.0 & 100.0 & 50.0 & 0.0 \\
\hline \multirow[t]{2}{*}{ Clarithromycin } & $\mathrm{S}$ & 0.0 & 0.0 & 0.0 & 0.0 & 0.0 & 0.0 & 0.0 & 0.0 & 0.0 & 0.0 & 0.0 \\
\hline & $\mathrm{R}$ & 0.0 & 0.0 & 0.0 & 0.0 & 0.0 & 0.0 & 0.0 & 0.0 & 100.0 & 0.0 & 0.0 \\
\hline \multirow[t]{2}{*}{ Tigecycline } & $\mathrm{S}$ & 100.0 & 100.0 & 100.0 & 100.0 & 75.0 & 100.0 & 0.0 & 0.0 & 0.0 & 100.0 & 0.0 \\
\hline & $\mathrm{R}$ & 0.0 & 0.0 & 0.0 & 0.0 & 25.0 & 0.0 & 0.0 & 0.0 & 0.0 & 0.0 & 0.0 \\
\hline \multirow[t]{2}{*}{ Nitrofurantoin } & $\mathrm{S}$ & 100.0 & 100.0 & 66.7 & 85.0 & 64.3 & 33.3 & 0.0 & 0.0 & 100.0 & 40.0 & 0.0 \\
\hline & $\mathrm{R}$ & 0.0 & 0.0 & 33.3 & 15.0 & 35.7 & 66.7 & 0.0 & 0.0 & 0.0 & 60.0 & 100.0 \\
\hline \multirow[t]{2}{*}{ Nalidixic Acid } & $\mathrm{S}$ & 0.0 & 0.0 & 50.0 & 25.0 & 0.0 & 0.0 & 0.0 & 0.0 & 0.0 & 0.0 & 100.0 \\
\hline & $\mathrm{R}$ & 0.0 & 0.0 & 50.0 & 75.0 & 100.0 & 0.0 & 0.0 & 100.0 & 0.0 & 100.0 & 0.0 \\
\hline \multirow[t]{2}{*}{ Azithromycin } & $\mathrm{S}$ & 0.0 & 25.0 & 0.0 & 50.0 & 70.0 & 100.0 & 0.0 & 100.0 & 0.0 & 50.0 & 0.0 \\
\hline & $\mathrm{R}$ & 100.0 & 75.0 & 0.0 & 50.0 & 30.0 & 0.0 & 0.0 & 0.0 & 0.0 & 50.0 & 0.0 \\
\hline \multirow[t]{2}{*}{ Erythromycin } & $\mathrm{S}$ & 0.0 & 0.0 & 0.0 & 100.0 & 0.0 & 0.0 & 0.0 & 0.0 & 0.0 & 0.0 & 0.0 \\
\hline & $\mathrm{R}$ & 0.0 & 0.0 & 0.0 & 0.0 & 0.0 & 0.0 & 0.0 & 0.0 & 0.0 & 0.0 & 0.0 \\
\hline \multirow[t]{2}{*}{ Chloramphenicol } & $\mathrm{S}$ & 0.0 & 0.0 & 0.0 & 66.7 & 0.0 & 0.0 & 0.0 & 100.0 & 0.0 & 100.0 & 0.0 \\
\hline & $\mathrm{R}$ & 0.0 & 0.0 & 0.0 & 33.3 & 0.0 & 0.0 & 100.0 & 0.0 & 0.0 & 0.0 & 0.0 \\
\hline \multirow[t]{2}{*}{ Pivmecillinam } & $\mathrm{S}$ & 0.0 & 0.0 & 0.0 & 0.0 & 0.0 & 0.0 & 0.0 & 0.0 & 0.0 & 0.0 & 0.0 \\
\hline & $\mathrm{R}$ & 0.0 & 0.0 & 0.0 & 0.0 & 0.0 & 0.0 & 0.0 & 0.0 & 0.0 & 0.0 & 0.0 \\
\hline Mecillinam & $\mathrm{S}$ & 0.0 & 0.0 & 0.0 & 0.0 & 0.0 & 0.0 & 0.0 & 0.0 & 0.0 & 0.0 & 0.0 \\
\hline & $\mathrm{R}$ & 0.0 & 0.0 & 0.0 & 100.0 & 0.0 & 0.0 & 0.0 & 0.0 & 0.0 & 0.0 & 0.0 \\
\hline Aztreonam & $\mathrm{S}$ & 0.0 & 0.0 & 0.0 & 0.0 & 0.0 & 0.0 & 0.0 & 0.0 & 0.0 & 100.0 & 0.0 \\
\hline & $\mathrm{R}$ & 0.0 & 0.0 & 0.0 & 100.0 & 0.0 & 0.0 & 0.0 & 0.0 & 0.0 & 0.0 & 0.0 \\
\hline Azactum & $\mathrm{S}$ & 33.3 & 100.0 & $0 .($ & 56.5 & 75.0 & 100.0 & 0.0 & 100.0 & 0.0 & 66.7 & 0.0 \\
\hline & $\mathrm{R}$ & 66.7 & 0.0 & 0. & 43.5 & 25.0 & 0.0 & 0.0 & 0.0 & 0.0 & 33.3 & 0.0 \\
\hline Linezolid & $\mathrm{S}$ & 100.0 & 100.0 & 0.0 & 88.9 & 100.0 & 0.0 & 0.0 & 0.0 & 100.0 & 100.0 & 0.0 \\
\hline & $\mathrm{R}$ & 0.0 & 0.0 & 0.0 & 11.1 & 0.0 & 0.0 & 0.0 & 0.0 & 0.0 & 0.0 & 0.0 \\
\hline Methicillin & $\mathrm{S}$ & 0.0 & 0.0 & 0.0 & 0.0 & 0.0 & 0.0 & 0.0 & 0.0 & 0.0 & 0.0 & 0.0 \\
\hline & $\mathrm{R}$ & 0.0 & 0.0 & 0.0 & 0.0 & 0.0 & 0.0 & 0.0 & 0.0 & 0.0 & 0.0 & 0.0 \\
\hline
\end{tabular}


Proteus was $100 \%$ sensitive to cephradine, cefoxitin, cefixime, ceftazidime, ceftriaxone, cefepime, cotrimoxazole, amikacin, ciprofloxacin, imipenem, meropenem, netilmicin, piperacillin+tazobactum combination, tetracycline, tigecycline, azithromycin, azactum and $100 \%$ resistant to doxycycline, tetracycline, chloramphenicol and cefuroxime.

Pseudomonas was $100 \%$ sensitive only to amikacin, netilmicin, and $100 \%$ resistant to cefixime, ceftazidime, ceftriaxone, cefepime, cotrimoxazole, gentamicin, ciprofloxacin, levofloxacin, imipenem, meropenem, doxycline, tetracycline, chloramphenicol.

Salmonella typhi was $100 \%$ sensitive to amoxicillin, cefoxitin, cefixime, ceftriaxone, cefepime, cotrimoxazole, amikacin, netilmicin, azithromycin, chloramphenicol, azactum and $100 \%$ resistant to cephradine, doxycycline, tetracycline, anlidixic acid.

MRSA was $100 \%$ sensitive to imipenem, vancomycin, teicoplanin, nitrofurantoin, linezolid and $100 \%$ resistant to cefpirome, cefoxitin, ceftazidime, cotrimoxazole, clindamycin, gentamicin, ciprofloxacin, netilmicin, tetracycline, clarithromycin.

Acinetobacter was $100 \%$ sensitive to penicillin, cefuroxime, colistin, piperacillin+tazobactum combination, tigecycline, chloramphenicol and $100 \%$ resistant to cefixime, nalidixic acid.

Citrobacter freundii was $100 \%$ sensitive to ceftazidime, ceftriaxone, cotrimoxazole, amikacin, gentamicin, ciprofloxacin, levofloxacin, imipenem, meropenem, netilmicin, nalidixic acid and $100 \%$ resistant to ampicillin, cefixime, nitrofurantoin.

\section{Discussion}

In our study, Enterococci were 100\% sensitive to piperacillin+tazobactum combination, tigecycline, nitrofurantoin and linezolid and $100 \%$ resistant to cefoxitin, cefixime, and moxifloxacin, azithromycin. Ahmed et.al. conducted a study to see the aerobic bacterial pattern in puerperal sepsis and found that all the isolates of Enterococcus were sensitive to amoxicillin and cephalexin. ${ }^{55}$
S. aureus was $100 \%$ sensitive to amikacin, moxifloxacin, imipenem, meropenem, piperacillin+tazobactum combination, vancomycin, doxycycline, tetracycline, tigecycline, nitrofurantoin, azactum, linezolid and $100 \%$ resistant to cefixime. Khan et.al. conducted a study to see prevalence of multidrug resistant Staphylococcus aureus isolates in clinical specimens collected from local patients of Chittagong, Bangladesh, and found that the rate of resistance against ampicillin, cephradine, gentamicin and ciprofloxacin were $92.1 \%, 60 \%, 58.1 \%$ and $59.35 \%$, respectively. ${ }^{56}$ Shahidullah et.al. found in a study to see the antibiotic sensitivity pattern of bacterial isolates from different clinical specimens at NICVD, Dhaka and found that Staphylococcus aureus was sensitive to only imipenem and cephalexin. ${ }^{40}$ Sultanan et.al. conducted a study to see the current microbial isolates from wound swab and their susceptibility pattern in a private medical college hospital in Dhaka city and found that Staphylococcus aureus was sensitive to linezolid $(94.38 \%)$, fusidic acid $(91.01 \%)$, vancomycin $(87.64 \%)$, amikacin $(74.15 \%)$ and gentamicin $(73.03 \%) .{ }^{48}$

In our study, Enterobacter was 100\% sensitive to penicillin, amikacin, gentamicin, netilmicin, doxycycline, tetracycline, tigecycline and $100 \%$ resistant to cefixime, ceftazidime, ceftriaxone, cefepime, cotrimoxazole, levofloxacin, vancomycin.

In our study, E. coli was $100 \%$ sensitive to imipenem, meropenem, vancomycin, tigecycline and $100 \%$ resistant to mecillinam, aztreonam. Kabir et.al. reported that enterotoxigenic E. coli were $100 \%$ sensitive to ceftriaxone, nitrofurantioin, amikacin, 94\% sensitive to nalidixic acid, $89 \%$ sensitive to gentamycin, $83 \%$ sensitive to ciprofloxacin, $79 \%$ sensitive to cephalexin, $39 \%$ sensitive to amoxycillin, $46 \%$ sensitive to tetracycline and $31 \%$ sensitive to cotrimoxazole. ${ }^{57}$ Begum et.al. analyzed 16,666 urine samples to see the trend of sensitivity pattern of uropathogenic Escherichia coli at Uttara Adhunik Medical College Hospital, in Dhaka. ${ }^{58}$ They found that A total number of 16, 666 reports of urine 
samples were collected from the microbiology laboratory data base of which 3,000(18\%) reports showed presence of E. coli. E. coli were mostly susceptible to meropenem from the year 2008 to 2012 (100\%) except 2010 (98.58\%) followed by amikacin $(81.20 \%-100 \%)$ and imipenem (78.66\%-100\%). Gradual decrease of susceptibility pattern of mecillinam was found. ${ }^{58}$

In our study, Klebsiella was $100 \%$ sensitive to flucloxacillin, colistin, vancomycin, tigecycline, linezolid and $100 \%$ resistant to nalidixic acid. Begum et.al. found in their study with neonatal sepsis patients, in NICU of BIRDEM, that Ampicillin and Gentamicin were 100\% resistant to Klebsiella third generation cephalosporin was also resistant to Klebsiella. Imipenem and meropenem were highly sensitive to all organisms. ${ }^{59}$

In our study, Proteus was $100 \%$ sensitive to cephradine, cefoxitin, cefixime, ceftazidime, ceftriaxone, cefepime, cotrimoxazole, amikacin, ciprofloxacin, imipenem, meropenem, netilmicin, piperacillin+tazobactum combination, tetracycline, tigecycline, azithromycin, azactum and $100 \%$ resistant to doxycycline, tetracycline, chloramphenicol and cefuroxime.

In our study, Pseudomonas was $100 \%$ sensitive only to amikacin, netilmicin, and 100\% resistant to cefixime, ceftazidime, ceftriaxone, cefepime, cotrimoxazole, gentamicin, ciprofloxacin, levofloxacin, imipenem, meropenem, doxycline, tetracycline, chloramphenicol. Ahmed et.al. conducted a study to see microbiological quality of street vended drinking water in Dhaka city and antibiotics resistance of isolated Salmonella spp and Pseudomonas spp. ${ }^{60}$ They found out that the Pseudomonas isolates showed a significant drug resistance to penicillin (100\%), ampicillin $(95 \%)$, amoxicillin $(95 \%)$ and nalidixic acid $(85 \%) .{ }^{60}$ Shahriar and Akter conducted a study to determine antimicrobial sensitivity of Pseudomonas aeruginosa isolated from clinical sources from different diagnostic centers, Dhaka, Bangladesh. ${ }^{61}$ They found very low sensitivity of $P$. aeruginosa towards cotrimoxazole $(45 \%)$, azithromycin $(30 \%)$ and erythromycin (35\%) was observed. Higher sensitivity pattern was observed for cefuroxime $(57.5 \%)$. and only imipenem $(100 \%)$ showed sensitivity pattern possibly susceptible enough to consider for the management of $P$. aeruginosa induced cases in the area under study. ${ }^{61}$ Shahidullah et.al., in their study to see the antibiotic sensitivity pattern of bacterial isolates from different clinical specimens at NICVD, Dhaka found that Pseudomonas species was resistant to penicillin, amoxycillin and vancomycin and $\sim 50 \%$ resistant to cotrimoxazole, cefuroxim, ceftriaxone, piperacillin, azythromycin, cephalexin, netelmycin and pfloxacillin. ${ }^{40}$

In our study, Salmonella typhi was $100 \%$ sensitive to amoxicillin, cefoxitin, cefixime, ceftriaxone, cefepime, cotrimoxazole, amikacin, netilmicin, azithromycin, chloramphenicol, azactum and $100 \%$ resistant to cephradine, doxycycline, tetracycline, nalidixic acid. Ahmed et.al., in their study to see conducted a study to see microbiological quality of street vended drinking water in Dhaka city and antibiotics resistance of isolated Salmonella spp and Pseudomonas spp, reported $100 \%$ of the Salmonella isolates were found resistant to penicillin. chloramphenicol, doxycycline, gentamycin, neomycin was sensitive to all of the isolates. ${ }^{60}$ Kawser et.al., in their study to see sensitivity pattern of azithrymycin, ofloxacin and ceftriaxone in ciprofloxacin resistant salmonella causing enteric fever, found that all ciprofloxacinresistant isolates were sensitive to ofloxacin (inhibitory zone diamater $16-32 \mathrm{~mm}$ ), ceftriaxone (inhibitory zone diameter $21 \mathrm{~mm}$ ), $66.66 \%$ isolates were sensitive to azithromycin. These results indicate that ofloxacin and ceflriaxone may be convenient alternative antimicrobial agents for Salmonella isolates. ${ }^{62}$ Nesa et.al. in their study to see the isolation, identification and characterization of Salmonella serovars from diarrhoeic stool samples of human The antimicrobial susceptibility testing showed that the isolated Salmonella serovars were highly sensitive to ciprofloxacin and moderately sensitive to chloramphenicol, kanamycin, cotrimoxazol and nalidixic acid. However, the 
positive isolates were resistant to erythromycin. ${ }^{63}$ Rahman MA, in his study, reported the resistance rates of $S$. typhi were $97.14 \%$ for cotrimoxazole, $95.29 \%$ for azithromycin, $91.43 \%$ for cefixime, $85.71 \%$ for tetracycline, $77.14 \%$ for ciprofloxacin and 68.57 $\%$ for ceftriaxone, respectively. Increased sensitivity was reported for imipenem $(88.57 \%)$, amikacin $(77.14 \%)$, chloramphenicol (65.71\%) and levofloxacin (42.86\%). ${ }^{64}$

In our study, MRSA was $100 \%$ sensitive to imipenem, vancomycin, teicoplanin, nitrofurantoin, linezolid and $100 \%$ resistant to cefpirome, cefoxitin, ceftazidime, cotrimoxazole, clindamycin, gentamicin, ciprofloxacin, netilmicin, tetracycline, clarithromycin. Shahriar MA reported in a study, out of 122 isolates, although no strains were found vancomycin resistant, $93.44 \%$ were found intermediate and only $6.56 \%$ showed sensitivity. ${ }^{65}$

In our study, Acinetobacter was 100\% sensitive to penicillin, cefuroxime, colistin, piperacillin+tazobactum combination, tigecycline, chloramphenicol and 100\% resistant to cefixime, nalidixic acid. Citrobacter freundii was $100 \%$ sensitive to ceftazidime, ceftriaxone, cotrimoxazole, amikacin, gentamicin, ciprofloxacin, levofloxacin, imipenem, meropenem, netilmicin, nalidixic acid and $100 \%$ resistant to ampicillin, cefixime, nitrofurantoin.

After the above discussion, and thorough review of the literature, it is evident that although some studies have been done in Bangladesh, the studies often have focused on a single pathogen, disease or body site. ${ }^{37-51}$ Moreover, modes and methods of presentation are so diverse, that it is very difficult to compare results of many studies. This article shows a comprehensive way of reporting both sensitivity and resistance of all organisms, in multiple specimens, and maybe useful for future research work to act like a template. In practice, we see more and more cases of resistance to broad spectrum antibiotics.

\section{Recommendations}

We thus propose following recommendations:

1. It should be routine practice to investigate the sensitivity pattern before prescribing antibiotics, and not to use broad spectrum antibiotics blindly.

2. To understand the always changing pattern of antibiotic sensitivity and resistance, studies should be conducted at regular intervals, and preferably should not be restricted to single specimen/bacterium species/body site.

3. Researchers should adopt a universal way of presentation of the findings to ensure easy comparability and understanding of findings.

4. The best approach should be to ensure all positive culture results be digitized and recorded at national level, so that prevalent bacteria, their susceptibility and resistance pattern for every locality can be known and decisions can be drawn to choose antimicrobial agents more wisely.

5. Doctors should be encouraged to order culture investigations before every prescription of antibiotics, and awareness should be raised among the patients and in the community about the need of this.

\section{Conclusion}

More and more antibiotics are becoming ineffective due to emergence of resistance. Serious actions should be taken. Awareness should be raised from the policy maker level to the physicians and patients.

Conflict of interest: None

\section{Funding: None}

\section{References}

1. Kunin CM. Resistance to antimicrobial drugs-a worldwide calamity. Ann Intern Med. 1993;118(7): 557-561.

2. Tomasz A. Multiple-antibiotic-resistant pathogenic bacteria-A report on the Rockefeller University workshop. N Engl J Med. 1994;330(17):12471251.

3. Baquero F, Blázquez J. Evolution of antibiotic resistance. Trends Ecol Evol. 1997;12(12):482487. 
4. Hart CA. Antibiotic resistance: an increasing problem? Br Med J. 1998;316(7140):1255-1257.

5. Hart CA, Kariuki S. Antimicrobial resistance in developing countries. BMJ. 1998;317(7159):647.

6. Pablos-Méndez A, Raviglione MC, Laszlo A, Binkin N, Rieder HL, Bustreo F, et al. Global surveillance for antituberculosis-drug resistance, 1994-1997. N Engl J Med. 1998;338(23):1641-1649.

7. Levy SB. Antibiotic resistance-the problem intensifies. Adv Drug Deliv Rev. 2005 Jul 29;57(10):1446-50.

8. Levy SB. Factors impacting on the problem of antibiotic resistance. J Antimicrob Chemother. $2002 ; 49(1): 25-30$.

9. Levy SB, Marshall B. Antibacterial resistance worldwide: causes, challenges and responses. Nat Med. 2004;10:S122-S129.

10. Su L-H, Chiu C-H, Chu C, Ou JT. Antimicrobial resistance in nontyphoid Salmonella serotypes: a global challenge. Clin Infect Dis. 2004; 39(4):546-551.

11. Cabello FC. Heavy use of prophylactic antibiotics in aquaculture: a growing problem for human and animal health and for the environment. Environ Microbiol. 2006;8(7):1137-1144.

12. Spellberg B, Guidos R, Gilbert D, Bradley J, Boucher HW, Scheld WM, et al. The epidemic of antibiotic-resistant infections: a call to action for the medical community from the Infectious Diseases Society of America. Clin Infect Dis. 2008;46(2):155-164.

13. Hawkey PM, Jones AM. The changing epidemiology of resistance. J Antimicrob Chemother. 2009;64(suppl 1):i3-i10.

14. Davies J, Davies D. Origins and evolution of antibiotic resistance. Microbiol Mol Biol Rev. 2010;74(3):417-433.

15. Rahman PMS. Antimicrobial Resistance: Global Context and Bangladesh Perspectives. [cited 2016 Dec 22]; Available from: http://www.academia. edu/1715842/ Antimicrobial_ Resistance_Global_ Context_and_Bangladesh__Perspectives

16. Levy SB. Antibiotic resistance: consequences of inaction. Clin Infect Dis. 2001;33(Supplement 3):S124-S129.

17. Arca P, Rico M, Braña AF, Villar CJ, Hardisson C, Suárez JE. Formation of an adduct between fosfomycin and glutathione: a new mechanism of antibiotic resistance in bacteria. Antimicrob Agents Chemother. 1988 Oct 1;32(10):1552-6.

18. Tomasz A, Drugeon HB, Lencastre HM de, Jabes D, McDougall L, Bille J. New mechanism for methicillin resistance in Staphylococcus aureus: clinical isolates that lack the PBP 2a gene and contain normal penicillin-binding proteins with modified penicillin-binding capacity. Antimicrob Agents Chemother. 1989 Nov 1;33(11):1869-74.

19. Dé E, Baslé A, Jaquinod M, Saint N, Malléa M, Molle G, et al. A new mechanism of antibiotic resistance in Enterobacteriaceae induced by a structural modification of the major porin. Mol Microbiol. 2001 Jul 1;41(1):189-98.

20. Murray BE, Mederski-Samaroj B. Transferable betalactamase. A new mechanism for in vitro penicillin resistance in Streptococcus faecalis. J Clin Invest. 1983 Sep;72(3):1168-71.

21. Ganguly NK, Arora NK, Chandy SJ, Fairoze MN, Gill JS, Gupta U, et al. Rationalizing antibiotic use to limit antibiotic resistance in India+. Indian J Med Res. 2011;134(3):281.

22. Rahman MM, Haq JA, Hossain MA, Sultana R, Islam F, Islam AHMS. Prevalence of extendedspectrum â-lactamase-producing Escherichia coli and Klebsiella pneumoniae in an urban hospital in Dhaka, Bangladesh. Int $\mathrm{J}$ Antimicrob Agents. 2004 Nov 1;24(5):508-10.

23. Mannan A, Shohel M, Rajia S, Mahmud NU, Kabir $\mathrm{S}$, Hasan I. A cross sectional study on antibiotic resistance pattern of Salmonella typhi clinical isolates from Bangladesh. Asian Pac J Trop Biomed. 2014 Apr;4(4):306-11.

24. Rashid M, Rakib MM, Hasan B. Antimicrobialresistant and ESBL-producing Escherichia coli in different ecological niches in Bangladesh. Infect Ecol Epidemiol [Internet]. 2015 Jul 17 [cited 2016 Dec 22];5(0). Available from: http:// www.infectionecologyandepidemiology.net/ index.php/iee/article/view/26712

25. Jahan N, Haque ZS, Mannan MA, Akhter M, Yasmin S, Akhter S, et al. Patient characteristics, Bacteriological profile \& outcome of Neonatal Sepsis: A Hospital Based Study. Community Based Med J. 2013 Mar;2(1):49â•"54.

26. Sutradhar KB, Saha A, Huda NH, Uddin R. Irrational Use of Antibiotics and Antibiotic Resistance in Southern Rural Bangladesh: Perspectives from Both the Physicians and Patients. ResearchGate [Internet]. 2014 Jan 15 [cited 2016 Dec 22];4(9). Available from: https:// ww w.research gate.net/publication / 259706314_Irrational_Use_of_Antibiotics_and_ Antibiotic_Resistance_in_ Southern Rural_Bangladesh_Perspectives_from Both_the_Physicians_and_Patients

27. Hossain MM, Glass RI, Khan MR. Antibiotic Use in a Rural Community in Bangladesh. Int $\mathrm{J}$ Epidemiol. 1982 Dec 1;11(4):402-5. 
28. Farrar WE. Antibiotic Resistance in Developing Countries. J Infect Dis. 1985;152(6):1103-6.

29. Biswas M, Roy MN, Manik MIN, Hossain MS, Tapu STA, Moniruzzaman M, et al. Self medicated antibiotics in Bangladesh: a cross-sectional health survey conducted in the Rajshahi City. BMC Public Health. 2014;14:847.

30. Fahad B, Matin A, Shil M, Asish K. Antibiotic usage at a primary health care unit in Bangladesh. Australas Med J. 2010;3(7):414-21.

31. Grüneberg RN. Antibiotic sensitivities of urinary pathogens, 1971-82. J Antimicrob Chemother. 1984 Jul 1;14(1):17-23.

32. Grüneberg RN. Changes in urinary pathogens and their antibiotic sensitivities, 1971-1992. J Antimicrob Chemother. 1994 May 1;33(suppl_A):1-8.

33. Yeo SG, Park DC, Hong SM, Cha CI, Kim MG. Bacteriology of chronic suppurative otitis media a multicenter study. Acta Otolaryngol (Stockh). 2007 Jan 1;127(10):1062-7.

34. Kahlmeter G. An international survey of the antimicrobial susceptibility of pathogens from uncomplicated urinary tract infections: the ECO-SENS Project. J Antimicrob Chemother. 2003 Jan 1;51(1):69-76.

35. Stoll BJ, Hansen N, Fanaroff AA, Wright LL, Carlo WA, Ehrenkranz RA, et al. Changes in Pathogens Causing Early-Onset Sepsis in Very-Low-BirthWeight Infants. N Engl J Med. 2002 Jul $25 ; 347(4): 240-7$

36. Lockhart SR, Abramson MA, Beekmann SE, Gallagher G, Riedel S, Diekema DJ, et al. Antimicrobial Resistance among Gram-Negative Bacilli Causing Infections in Intensive Care Unit Patients in the United States between 1993 and 2004. J Clin Microbiol. 2007 Oct 1;45(10):33529 .

37. Chowdhury MK, Siddique AA, Sarkar PK, Haque $\mathrm{MM}$, Biswas PK, Biswas S, et al. Pattern of Antibiotic Use in Different Departments of Dhaka Medical College Hospital. J Med. 2015 Feb $25 ; 16(1): 35-8$.

38. Chowdhury FFK, Ahsan S, Kabir MS. Antibiotic resistance patterns of pathogenic Gram negative bacteria isolated from UTI patients in Sirajganj district. Stamford J Microbiol. 2015 Mar 26;3(1):17-20.

39. Nawas T, Mazumdar RM, Das S, Nipa MN, Islam S, Bhuiyan HR, et al. Microbiological Quality and Antibiogram of E. coli, Salmonella and Vibrio of Salad and Water from Restaurants of Chittagong. J Environ Sci Nat Resour. 2012 Aug 7;5(1): 159-66.
40. Shahidullah MS, Yusuf MA, Khatun Z, Ara U, Mitul MT. Antibiotic Sensitivity Pattern of Bacterial Isolates from Different Clinical Specimens: Experience at NICVD, Dhaka. Cardiovasc J. 2012 Oct 19;5(1):67-72.

41. Asna SMZH, Akhter S, Rahman MM, Mohammad N, Hafez MA. Frequency and Sensitivity of Extended Spectrum Beta-Lactamase Positive Organisms in a Secondary and Tertiary Level Hospital Network in Dhaka. J Enam Med Coll. 2015 Jun 29;5(2):80-7.

42. Biswas R, Rabbani R, Ahmed HS, Sarker MAS, Zafrin N, Rahman MM. Antibiotic sensitivity pattern of urinary tract infection at a tertiary care hospital. Bangladesh Crit Care J. 2014 Aug $11 ; 2(1): 21-4$.

43. Rahman M, Islam MN, Islam MN, Hossain MS. Isolation and Identification of Oral Bacteria and Characterization for Bacteriocin Production and Antimicrobial Sensitivity. Dhaka Univ J Pharm Sci. 2015 Jun 18;14(1):103-9.

44. Jhora ST, Paul S. Urinary Tract Infections Caused by Staphylococcus saprophyticus and their antimicrobial sensitivity pattern in Young Adult Women. Bangladesh J Med Microbiol. 2011 Jan 24;5(1):21-5.

45. Akter J, Khan JG, Khan MH, Hossain MZ. Psychological Distress in Cancer Patients with Underage Children. J Dhaka Med Coll. 2016 Sep $15 ; 24(2): 146-51$.

46. Sultana S, Khatun M, Ali MK, Mawla N, Akhter N. Pattern of antibiotic sensitivity of bacteria causing urinary tract infection in a private medical college hospital in Dhaka. Bangladesh J Med Sci. 2015 Jan 10;14(1):70-4.

47. Nusrat F, Islam KMS, Shamsuzzaman SM, Yusuf MA, Farzana A, Afrin S. Frequency and Sensitivity Pattern of Neisseria gonorrhoae Isolated from Women at a Tertiary Care Hospital in Dhaka City. Bangladesh J Infect Dis. 2015 Jan 6;1(1):3-7.

48. Sultana S, Mawla N, Kawser S, Akhtar N, Ali MK. Current Microbial Isolates from Wound Swab and Their Susceptibility Pattern in a Private Medical College Hospital in Dhaka city. Delta Med Coll J. 2015 Feb 14;3(1):25-30.

49. Mondal MK, Roy BR, Yeasmeen S, Haque F, Huda $\mathrm{AQ}$, Banik D. Prevalence of microorganism and emergence of bacterial resistance in ICU of Bangabandhu Sheikh Mujib Medical University of Bangladesh. J Bangladesh Soc Anaesthesiol. 2014 Aug 3;26(1):20-6.

50. Afroze SR, Rahim MA, Hasan MM, Afroz F, Haque HF, Ahmed JU, et al. Pattern of Antibiotic Sensitivity in Enteric Fever: A Tertiary Care Hospital Experience. J Med. 2014 Oct 19;15(2):122-4. 
51. Yasmeen BN, Islam S, Islam S, Uddin MM, Jahan R. Prevalence of urinary tract infection, its causative agents and antibiotic sensitivity pattern/ : A study in Northern International Medical College Hospital, Dhaka. North Int Med Coll J. 2015 Nov 16;7(1):105-9.

52. Levinson W. Review of Medical Microbiology and Immunology. 14 edition. USA: McGraw-Hill Education / Medical; 2016. 61-68 of 832 p.

53. Drew WL. Value of sputum culture in diagnosis of pneumococcal pneumonia. J Clin Microbiol. 1977 Jul 1;6(1):62-5.

54. Bauer AW, Kirby WM, Sherris JC, Turck M. Antibiotic susceptibility testing by a standardized single disk method. Am J Clin Pathol. 1966 Apr;45(4):493-6.

55. Ahmed S, Hossain MA, Shamsuzzaman AKM, Sumona AA, Bagum Z, Jahan NA, et al. Aerobic Bacterial Pattern in Puerperal Sepsis. Bangladesh J Med Microbiol. 2016 May 25;2(1):22-7.

56. Khan SU, Mahmud MN, Chowdhury MA, Hakim MA. Prevalence of Multidrug resistant Staphylococcus Aureus isolates in Clinical specimens collected from local patients of Chittagong, Bangladesh. Chittagong Univ J Biol Sci. 2013 Dec 2;6(1-2):175-85.

57. Kabir MR, Hossain MA, Alam MM, Paul SK, Begum $Z$, Parvin US, et al. Frequency and Antimicrobial Susceptibility of Diarrheagenic Escherichia coli Obtained from Patients with Acute Diarrhea in a Tertiary Care Hospital, Bangladesh. Community Based Med J. 2013 Oct 28;2(2):46-51.

58. Begum MF, Mosaddek ASM, Perveen K, Karim R, Begum NN. Trend of Sensitivity Pattern of Uropathogenic Escherichia coli: Five Year Experience at a Tertiary Care Hospital in Dhaka. J Shaheed Suhrawardy Med Coll. 2013 Dec $1 ; 5(2): 103-5$.

59. Begum S, Baki MA, Kundu GK, Islam I, Kumar M, Haque A. Bacteriological Profile of Neonatal Sepsis in a Tertiary Hospital in Bangladesh. J
Bangladesh Coll Physicians Surg. 2012 Jul 23;30(2):66-70.

60. Ahmed S, Mahbub KR, Ahmed MM, Rahman M, Hoque MM. Microbiological Quality of Street Vended Drinking Water in Dhaka City and Screening for Antibiotics Resistance of Isolated Salmonella spp and Pseudomonas spp. J Sci Res. 2014 Apr 24;6(2):359-71.

61. Shahriar M, Akter S. A Survey on Antimicrobial Sensitivity Pattern of Different Antibiotics on Clinical Isolates of Pseudomonas aeruginosa Collected from Dhaka City of Bangladesh. J Sci Res. 2010 Dec 20;3(1):187.

62. Kawser S, Miah RA, Sabah KMN, Begum T, Sultana S. Sensitivity pattern of azithrymycin, ofloxacin and ceftriaxone in ciprofloxacin resistant salmonella causing enteric fever. J Dhaka Med Coll [Internet]. 2013 Jul 8 [cited 2017 Apr 12];22(1). Available from: http:// www.banglajol.info/index.php/JDMC/article/ view / 15627

63. Nesa M, Khan M, Alam M. ISOLATION, IDENTIFICATION AND CHARACTERIZATION OF SALMONELLA SEROVARS FROM DIARRHOEIC STOOL SAMPLES OF HUMAN. Bangladesh J Vet Med [Internet]. 2012 Jul 13 [cited 2017 Apr 12];9(1). Available from: http://www.banglajol. info/index.php/BJVM/article/view/11218

64. Rahman MA. Antimicrobial Resistance Patterns of Salmonella Typhi Isolated from Stool Culture. Chattagram Maa-O-Shishu Hosp Med Coll J [Internet]. 2015 Apr 4 [cited 2017 Apr 12];14(1). Available from: http://www.banglajol.info/index. php/CMOSHMCJ/article/view/22876

65. Shahriar M, Shahid S, Katha KK, Nasreen W, Bhuiyan MA. Vancomycin Sensitivity of Clinical Isolates of Staphylococcus aureus from Patients in Dhaka City, Bangladesh. Bangladesh Pharm J [Internet]. 2012 Nov 12 [cited 2017 Apr 12];15(2). Available from: http://www.banglajol.info/ index.php/BPJ/article/view/ 12582 